Research Article

\title{
Estimating the Ground and Excited State Dipole Moments of Levofloxacin and Norfloxacin Drugs Using Solvatochromic Effects and Computational Work
}

\author{
Kinfe Woldegiorges $(\mathbb{D}$, Abebe Belay $(D)$, Alemu Kebede, and Tamirat Abebe $(\mathbb{D}$ \\ Department of Applied Physics, School of Applied Natural Sciences, Adama Science and Technology University, PO Box 1888, \\ Adama, Ethiopia
}

Correspondence should be addressed to Kinfe Woldegiorges; kinfebwgio@gmail.com and Abebe Belay; abebebelay96@gmail.com

Received 28 May 2021; Revised 6 July 2021; Accepted 26 November 2021; Published 10 December 2021

Academic Editor: Jose M. Pedrosa

Copyright (c) 2021 Kinfe Woldegiorges et al. This is an open access article distributed under the Creative Commons Attribution License, which permits unrestricted use, distribution, and reproduction in any medium, provided the original work is properly cited.

Levofloxacin (LVF) and norfloxacin (NRF) are a group of fluoroquinolone antibiotics, broad spectrum used to treat various infections caused by many bacterial species. The drugs contain functional groups which control the type and degree of interaction with different solvents. In this research, the ground and excited state dipole moments of LVF and NRF drugs were estimated using solvatochromic effects and computational work. The dipole moments were estimated from absorption and emission spectra in polar and nonpolar solvents using Bakhshiev's, Kawski-Chamma-Viallet, Lippert-Mataga, and Reichardt models. The results indicated the emission spectra are more strongly affected by solvent polarity than the absorption spectra. The calculated excited state dipole moment is larger than that of the ground state, indicating that the probe compounds are significantly more polarized in the excited state than in the ground state. From computational work, the HOMO-LUMO energy band gap, the dipole moments, electron charge density distribution, and oscillator strength were determined using the semiempirical MP6 method, DFT-B3LYP6-31G, and DFT-B3LYP-3-21G employing Gaussian 09 software. In general, larger dipole moments were obtained by computation rather than from experiments due to the absence of solvent effects.

\section{Introduction}

Levofloxacin (LVF) and norfloxacin (NRF) are a group of fluoroquinolone antibiotics with a broad medicinal spectrum, active against Gram-negative and Gram-positive bacteria. Figures $1(\mathrm{a})$ and $1(\mathrm{~b})$ are the chemical structure of NRF and LVF. The drugs contain several proton binding sites such as carboxyl, carbonyl, and amino groups [1]. Different studies indicated that the polarity parameters of a drug originated from its chemical structure and functional groups attached to the compound [2]. Due to these properties, the physical and chemical behaviors of the drugs are altered in solvents $[3,4]$. The functional groups that exist in drugs are controlling the type and degree of interaction with solvents $[5,6]$. Also, it was observed that due to these functional groups, the antibacterial activity of the drugs is
$\mathrm{pH}$ dependent $[1,7]$. Hence, studying the interaction of solvents with drugs is important for biological applications and to get information about change in electronic distribution upon excitation [8].

Recently, the effects of solvent media on the photophysical properties of different drug, such as vitamin A [9], vitamin B [10], triarylmethane [11], folic acid [12], and folate derivatives [13], were investigated using different spectroscopic techniques. The results of the study indicated that the general solvent effect due to relative permittivity and refractive index and specific due to hydrogen bonding and intermolecular charge transfer were observed between the drugs and solvents. As the solvents polarity changes, shifts of the absorption and emission peaks are observed and result in change in the dipole moments due to the effect of the solvent's polarity $[14,15]$. Estimating the ground and excited 
<smiles>C[C@H]1COc2c(N3CCN(C)CC3)c(F)cc3c(=O)c(C(=O)O)cn1c23</smiles>

(a)<smiles>CCN1C=C(C(=O)O)C(=O)C2C=C(F)C(N3CCNCC3)=CC21</smiles>

(b)

Figure 1: Molecular structure of (a) LVF and (b) NRF.

state dipole moments of drugs from solvatochromic effects and computational work has great importance to reveal information on the electronic and geometrical structures of these drug molecules [2, 16]. It also reflects the charge distribution in the molecule and is useful in parameterization in quantum chemical procedures [17]. The biological activities of the molecules are mainly depending on their molecular structures. This can be obtained from ground and excited state dipole moments. A small change of the dipole moments and molecular structure may cause different biological activities related to the drug, making the dipole moments important measurable properties of drugs [18].

Although the photophysical properties of some vitamins and other drugs were studied, however, to the best of our knowledge, the solvatochromic effect of LVF and NRF in polar and nonpolar solvents for the determination of ground and excited state dipole moments have not been investigated experimentally and theoretically. Therefore, in this research the ground and excited state dipole moment are estimated experimentally using Lippert-Mataga, Bakhshiev's, Kawski-Chamma-Viallet, and Reichardt equations and computational work using DFT and semiempirical methods employing Gaussian 09 software.

\section{Theoretical Background}

To determine the ground and excited state dipole moments of a molecule by solvatochromic method the equation relating the difference and sum of absorption $\left(v_{a}\right)$ and fluorescence $\left(v_{f}\right)$, wavenumbers to solvent polarity functions are given by (1)-(3) [19-23].

Lippert-Mataga equation is as follows [21, 22]:

$$
v_{a}-v_{f}=m f\left(\varepsilon_{r}, n\right)+\text { const. }
$$

Bakhshiev's equation is as follows [23]:

$$
v_{a}-v_{f}=m_{1} f\left(\varepsilon_{r}, n\right)+\text { const. }
$$

Kawski-Chamma-Viallet equation is as follows [19, 20]:

$$
v_{a}+v_{f}=-m_{2} f\left(\varepsilon_{r}, n\right)+2 g(n)+\text { const, }
$$

Where $f\left(\varepsilon_{r}, n\right)$ and $g(n)$ are the solvent polarity functions, dependent on the dielectric constant $\varepsilon_{\mathrm{r}}$ and the index of refraction $n$. Parameters $m, m_{1}$, and $m_{2}$ are determined from the slopes of (1), (2), and (3), respectively, and are related to ground and excited state dipole moment using the following equations:

$$
\begin{aligned}
& m=\frac{2\left(\mu_{e}-\mu_{g}\right)^{2}}{h c a^{3}}, \\
& m_{1}=\frac{2\left(\mu_{e}-\mu_{g}\right)^{2}}{h c a^{3}}, \\
& m_{2}=\frac{2\left(\mu_{e}^{2}-\mu_{g}^{2}\right)}{h c a^{3}} .
\end{aligned}
$$

The parameters $\mu_{e}$ and $\mu_{q}$ are the excited and ground state dipole moment of the solute molecule, $c$ is the speed of light in vacuum, $h$ is Planck's constant, and $a$ is Onsager cavity radius of the solute molecule and is determined using the following equation $[24,25]$ :

$$
a=\left(\frac{3 M}{4 \pi \delta N}\right)^{1 / 3}
$$

where $\mathrm{M}$ is the relative molecular mass of the solute molecules, $\mathrm{N}$ is Avogadro's number, and $\delta$ is the density assuming that the molecules are spherical. The solvent polarity function used in the Lippert-Mataga equation is described in the following [21]:

$$
f\left(\varepsilon_{r}, n\right)=\frac{\varepsilon_{r}-1}{2 \varepsilon_{r}+2}-\frac{n^{2}-1}{2 n^{2}+1} .
$$

Substituting (8) into (1), the Lippert-Mataga equation is obtained [22]:

$$
v_{a}-v_{f}=m\left(\frac{\varepsilon_{r}-1}{2 \varepsilon_{r}+2}-\frac{n^{2}-1}{2 n^{2}+1}\right)+\text { const. }
$$

From the slope $m$ of the graph of $v_{a}-v_{f}$ versus Lippert-Mataga solvent polarity function, change in the dipole moment is expressed as follows:

$$
\Delta \mu=\mu_{e}-\mu_{g}=\left(\frac{m h c a^{3}}{2}\right)^{1 / 2} .
$$

The solvent polarity functions used in Bakhshiev's and Kawski-Chamma-Viallet equation are expressed in the following equations according to $[19,20]$ : 


$$
\begin{aligned}
f\left(\varepsilon_{r}, n\right) & =\frac{2 n^{2}+1}{2\left(n^{2}+2\right)}\left(\frac{\varepsilon_{r}-1}{\varepsilon_{r}+2}-\frac{n^{2}-1}{n^{2}+2}\right), \\
g(n) & =\frac{3}{2}\left(\frac{n^{4}-1}{\left(n^{2}+2\right)^{2}}\right) .
\end{aligned}
$$

When (11) is substituted into (2), we will get Bakhshiev's equation [23]:

$$
v_{a}-v_{f}=m_{1} \frac{2 n^{2}+1}{n^{2}+2}\left(\frac{\varepsilon_{r}-1}{\varepsilon_{r}+2}-\frac{n^{2}-1}{n^{2}+2}\right)+\text { const. }
$$

Similarly, when (11) and (12) are substituted into (3), we can get the Kawski-Chamma-Viallet equation [20]:

$$
\begin{aligned}
v_{a}+v_{f}= & -m_{2}\left(\frac{2 n^{2}+1}{n^{2}+2}\left(\frac{\varepsilon_{r}-1}{\varepsilon_{r}+2}-\frac{n^{2}-1}{n^{2}+2}\right)\right) \\
& +3\left(\frac{n^{4}-1}{\left(n^{2}+2\right)^{2}}\right)+\text { const. }
\end{aligned}
$$

If the symmetry of the investigated solute molecule remains unchanged during electron excitation, the following expressions are obtained:

$$
\begin{aligned}
& \mu_{g}=\frac{\left|m_{2}-m_{1}\right|}{2}\left(\frac{h c a^{3}}{2 m_{1}}\right)^{1 / 2}, \\
& \mu_{e}=\frac{\left|m_{2}+m_{1}\right|}{2}\left(\frac{h c a^{3}}{2 m_{1}}\right)^{1 / 2}, \\
& \frac{\mu_{e}}{\mu_{g}}=\frac{\left|m_{2}+m_{1}\right|}{\left|m_{2}-m_{1}\right|} \mu_{g}, \quad \text { for }\left(m_{2}>m_{1}\right) .
\end{aligned}
$$

The dipole moments can also be determined using another method that is based on the empirical solvent polarity scale $\left(E_{T}^{N}\right)$. The idea was initially expressed by Reichardt [26] and developed by Ravi [27]. The method is based on solvatochromic properties of betaine dye, correlated with the polarization and hydrogen bonding effect, and is expressed as follows:

$$
v_{a}-v_{f}=11307.6\left[\left(\frac{\Delta \mu}{\Delta \mu_{B}}\right)^{2}\left(\frac{a_{B}}{a}\right)^{3}\right] E_{T}^{N}+\text { const }
$$

where $\Delta \mu_{B}=9$ Debye is the change in dipole moment, $a_{B}=$ $6.2 A$ is the Onsager radius for betaine dye, $\Delta \mu$ and $a$ are the corresponding quantities for molecule of interest, and $\left(E_{T}^{N}\right)$ is given by

$$
E_{T}^{N}=\frac{E_{T}(30)_{\text {solvent }}-E_{T}(30)_{\mathrm{TMs}}}{E_{T}(30)_{\text {water }}-E_{T}(30)_{\mathrm{TMs}}}=\frac{E_{T}(30)_{\text {solvent }}-30.7}{32.4} .
$$

In this case, TMS represents tetramethylsilane known as a nonpolar solvent $\left(E_{T}^{N}=0\right)$ and using water as a highly polar solvent $\left(E_{T}^{N}=1\right)$. The change in dipole moment is determined from the slope of the linear plot of $v_{a}-v_{f}$ versus $E_{T}^{N}$ of (16) described as follows:

$$
\Delta \mu=\mu_{e}-\mu_{g}=\sqrt{\frac{m \times 81}{(6.2 / a)^{3} 11307.6}} .
$$

\section{Materials and Methods}

3.1. Experimental. LVF and NRF drugs were purchased from Sigma-Aldrich Company and used without further purification. The polar solvents (distilled water, methanol, ethanol, ethyl glycol, and ethyl acetate) and non-olar solvents (chloroform, dichloromethane, and isopropanol) used are all spectroscopic grade. The absorption spectra of the drugs were measured by double beam UV/Vis spectrophotometry (an ISO 9001 model, Maalab, India) in the wavelength region of 200-400 nm using $1 \mathrm{~cm}$ quartz cuvettes at room temperature. The steady state fluorescence emission spectra were measured using the Cary Eclipse Fluorescence Spectrophotometer (Agilent, Malaysia). The excitation wavelength and emission spectra were measured at $290 \mathrm{~nm}$ and 350-600 nm, respectively. The excitation and emission slit width is set at $10 \mathrm{~nm}$.

Stock solution of $2 \times 10^{-4} \mathrm{M}$ LVF and NRF were prepared in polar and nonpolar solvents and stored in the refrigerator to protect the samples from sunlight. The $\mathrm{UV} / \mathrm{V}$ is absorption and steady state fluorescence emission measurements were performed at room temperature using low concentrations: absorbance $<1$ au for absorption and absorbance $<0.1$ au for the fluorescence spectra measurement. The absorption and emission spectra were analysed using Origin 8 software. The values of the solvent polarity functions were calculated from relative permittivity, refractive index of the solvents, and empirical solvent polarity parameters as shown in Table 1.

3.2. Computational Method. To understand the electronic structure and electronic properties of LVF and NRF, computational work was performed employing Gaussian 09 software. The HOMO-LUMO energy band gap, the dipole moments, electron charge density distribution, oscillator strength, and electrostatic potential of the molecules were computed using semiempirical methods PM6, DFT-B3LYP6-31G, and 21G, respectively. Time-dependent DFT (TDDFT) with basis set 6-31G is used to calculate the excited state dipole moment. All the calculations were performed after optimizing the geometry of the molecule in the ground state [28].

\section{Results and Discussion}

4.1. Effects of Solvent Polarity on Absorption and Emission Spectra of Levofloxacin and Norfloxacin Drugs. Absorption and emission spectra of molecules in solvents provide reliable information about solvation effects on the ground and excited states [29]. Figures 2(a), 2(b), 3(a), and 3 (b) are the absorption and fluorescence emission spectra of LVF and NRF in different polar and nonpolar solvents, respectively. Two absorption peaks were observed for LVF with the highest peak band at 275-325 nm and weak band at 
TABLE 1: Calculated values of solvent polarity functions in different solvents.

\begin{tabular}{lccccc}
\hline Solvents & $n$ & $\varepsilon_{r}$ & $f_{B a c}\left(\varepsilon_{r}, n\right)$ & $f_{K C V}\left(\varepsilon_{r}, n\right)+2 g(n)$ & $f_{L M}\left(\varepsilon_{r}, n\right)$ \\
\hline Chloroform & 1.445 & 4.81 & 0.3714 & 0.9634 & 0.1486 \\
Dichloromethane & 1.424 & 8.93 & 0.4745 & 1.0295 & 0.203 \\
Isopropanol & 1.3776 & 19.9 & 0.7786 & 1.2923 & 0.253 \\
Ethanol & 1.3616 & 24.5 & 0.8126 & 1.3348 & 0.2762 \\
Methanol & 1.33 & 32.7 & 0.8542 & 1.3041 & 0.2886 \\
Distilled water & 1.3325 & 80.1 & 0.9138 & 1.3636 & 0.346 \\
Ethyl acetate & 1.372 & 6.02 & 0.4895 & 0.9955 & 0.654 \\
Ethyl glycol & 1.4382 & 37.7 & 0.8394 & 1.4334 & 0.762 \\
\hline
\end{tabular}

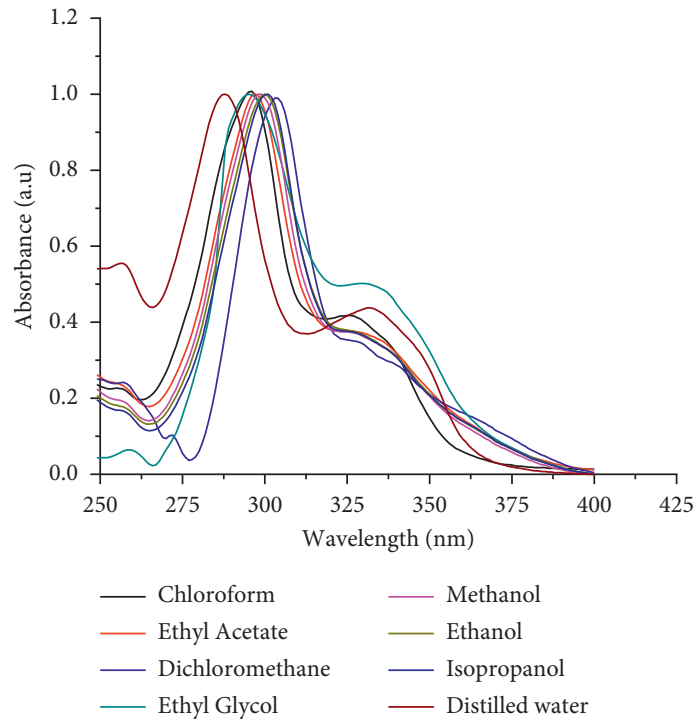

(a)

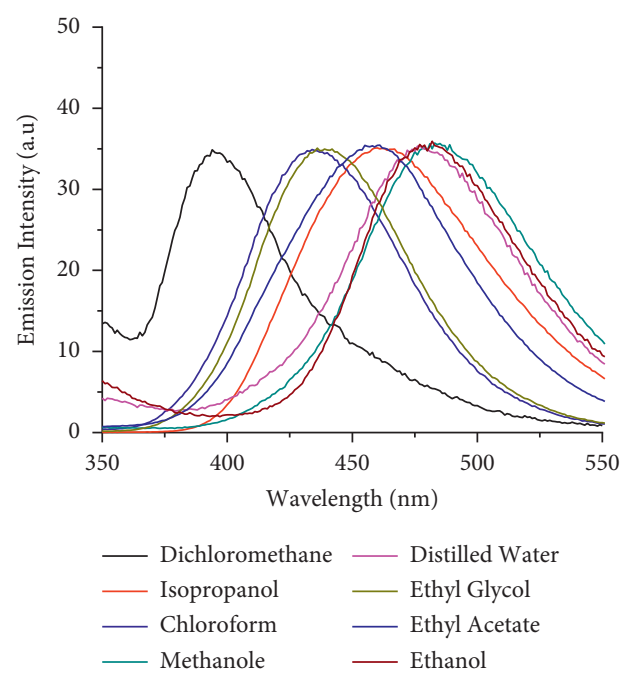

(b)

FIGURE 2: (a) UV/Vis absorption spectra and (b) emission spectra of LVF in solvents with different solvent polarities.

325-350 nm, respectively. Similarly for NRF, the main peak band appeared at $250-300 \mathrm{~nm}$ and weak band at 300-350 nm. Generally, the absorption spectra of the two drugs showed a blue shift with increasing solvent polarity. This is because the increase of solvent polarity causes a blue shift in the $n \longrightarrow \pi^{*}$ absorption of carbonyl compounds [30]. In addition, $n$ state is more easily stabilized by polar solvent effects such as hydrogen bonding, so in going from nonpolar solvent to polar solvent, there is a blue shift.

On the other hand, the emission spectra of the drugs show red shifts with increasing solvent polarity, and these are due to the fact that the excited states of LVF and NRF are more stabilized in polar solvent than nonpolar solvents [30, 31]. The largest peak emission shifts in different solvents are $44 \mathrm{~nm}$ and $39 \mathrm{~nm}$ for LVF and NRF, respectively, as shown in Table 2. Larger shifts of the emission spectra when compared to the shifts of the absorption were observed. The reason is that absorption of light occurs in about $10^{-15} \mathrm{~s}$, a time too short for motion of the fluorophore or solvent. Hence, the absorption spectra are less sensitive to solvent polarity. In contrast, the emitting fluorophore is exposed to the relaxed environment, which contains solvent molecules oriented around the dipole moment of the excited state [11]. In general, a large emission shift shows that the excited state geometry of the compounds is different from the ground state geometry and that the dipole moment increases during excitation. As shown in Table 2, the smallest and largest values of the wavenumbers of the emission peaks of LVF and NRF were observed in water (polar solvent) and dichloromethane (nonpolar solvent), respectively. The results indicated that both LVF and NRF have strong intermolecular interaction with polar solvents in the excited state.

Large Stokes shifts were observed for both LVF and NRF antibiotic drugs and this can be an indication of intramolecular charge transfer (ICT) occurring as result of excitation [32, 33]. Previous work confirms that large Stokes shift seen on fluoroquinolones antibiotic drugs in aqueous solution is explained through intramolecular charge transfer from the (N1) piperazinyl group to the main ring of the molecule [34].

4.2. Evaluation of Dipole Moments. In the excited state, the dipole moment of the molecule is changed due to redistribution of electron density relative to ground state. The change of the dipole moment is influenced the nature of the surrounding media/solvent and the type of solute-solvent interactions [35]. To understand the solvatochromic effects of LVF and NRF, the absorption and emission spectra are correlated to solvent polarity functions and empirical solvent polarity parameter, and thus ground and excited state 


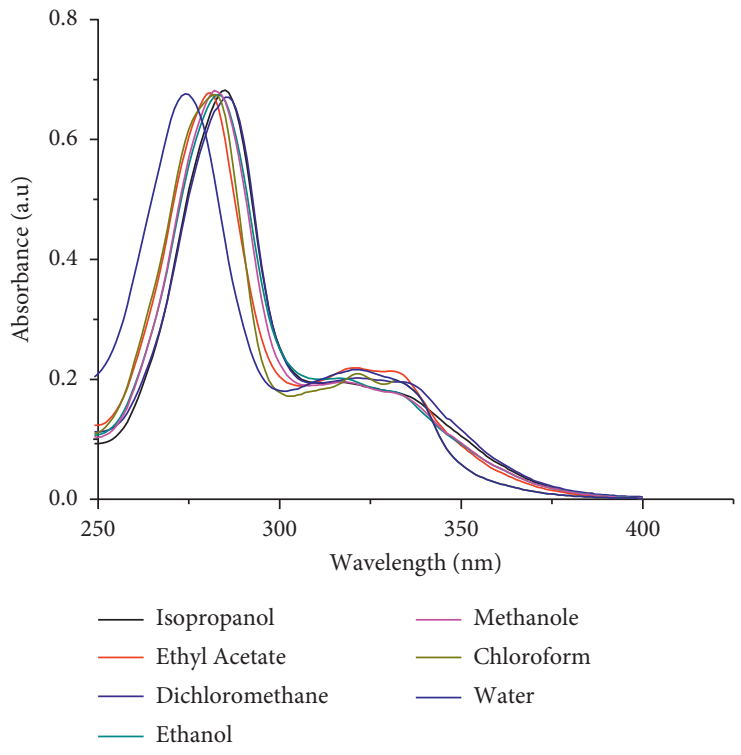

(a)

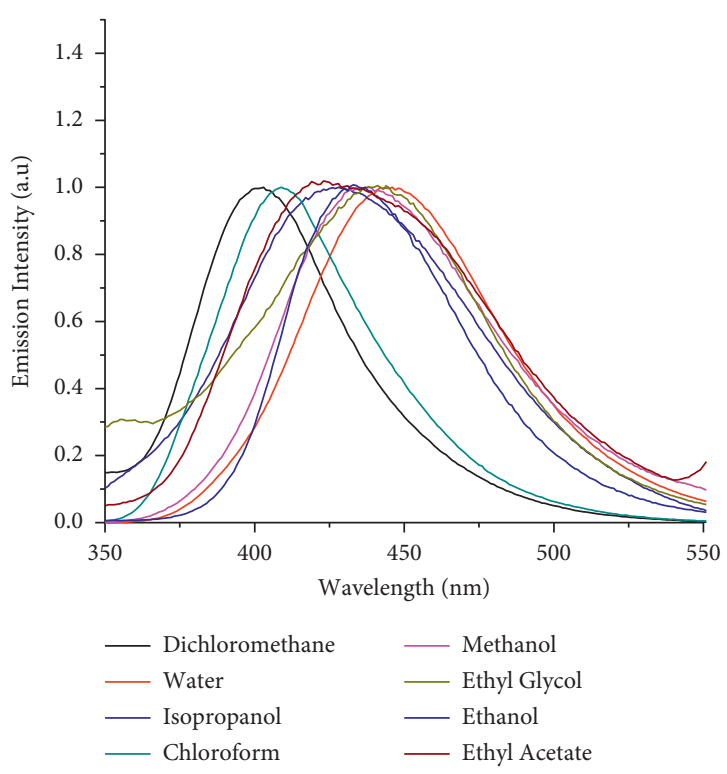

(b)

FIGURE 3: (a) UV/Vis absorption spectra and (b) emission spectra of NRF in solvents with different solvent polarities.

TABLE 2: Peaks of the absorption and emission spectra of LVF and $\mathrm{NRF}$ in solvents with different polarity.

\begin{tabular}{lcccc}
\hline \multirow{2}{*}{ Solvent } & \multicolumn{2}{c}{ Norfloxacin } & \multicolumn{2}{c}{ Levofloxacin } \\
& $v_{a}\left(\mathrm{~cm}^{-1}\right)$ & $v_{f}\left(\mathrm{~cm}^{-1}\right)$ & $v_{a}\left(\mathrm{~cm}^{-1}\right)$ & $v_{f}\left(\mathrm{~cm}^{-1}\right)$ \\
\hline Chloroform & 35460.99 & 24626.9 & 33783.78 & 22782.68 \\
Dichloromethane & 34965.03 & 24812.05 & 32894.74 & 22373.37 \\
Isopropanol & 35087.72 & 23588.8 & 33222.59 & 20966.12 \\
Ethanol & 35335.69 & 23093.09 & 33222.59 & 20788.29 \\
Methanol & 35460.99 & 22885.39 & 33444.82 & 20704.79 \\
Distilled water & 36496.35 & 22727.27 & 34722.22 & 21415 \\
Ethyl acetate & 34722.22 & 24862.36 & 33444.82 & 21689.15 \\
Ethyl glycol & 34602.08 & 22672.07 & 33898.3 & 20833.33 \\
\hline
\end{tabular}

dipole moments of LVF and NRF were estimated from the slope of the Lippert-Mataga equation (9), Bakhshiev's equation (13), the Kawski-Chamma-Viallet equation (14), and the Reichardt equation (18) fitted to the experimental data. The results are shown in Table 3. Figures 4(a) and 4(b) are the graphs of $v_{a}-v_{f}$ versus $f\left(\varepsilon_{r}, n\right)$ for LVF and NRF using Bakhshiev's equation. The statistical analysis of the graphs for both drugs has good linearity with high correlation coefficients. Similarly, Figures 5(a) and 5(b) are the graphs of $v_{a}+v_{f}$ versus $f\left(\varepsilon_{r}, n\right)+2 g(n)$ using KawskiChamma-Viallet equation with good correlation coefficients. The slopes, intercepts, and correlation coefficients of LVF and NRF are summarized in Table 3. In some cases, deviation of data point from linearity were observed in the graph $v_{a}-v_{f}$ versus $f\left(\varepsilon_{r}, n\right)$ and $v_{a}+v_{f}$ versus $f\left(\varepsilon_{r}, n\right)+2 g(n)$. This is probably due to specific solutesolvent interactions (e.g., hydrogen bond formation) that are not taken into consideration in all the above-mentioned theories, and it indicates the extent of interactions between the solute and solvent molecules [36-38].

From the slope of the graphs and using (15) and (16), the ground and excited state dipole moments of LVF and NRF were determined, and the results are depicted in Table 4 . The calculated results indicate that the excited state dipole moment is larger than the ground state dipole moment indicating that the probe compounds are significantly more polarized in the excited state than in the ground state. The ground and excited state dipole moments that are estimated by different methods are not similar due to the fact that different assumption and simplification are applied in each method [39]. It is also noted that the values of the ground and excited state dipole moments of LVF and NRF are not similar. The variation in the value of dipole moment may be due to structural difference of the two compounds [40]. In addition, the change in dipole moments $(\Delta \mu=13.6,16.4 \mathrm{D})$ obtained using Lippert-Mataga equation (9) are larger than the value obtained by other methods. This is due to the fact that Lippert-Mataga equation neglected polarizability of the solute molecules [41]. Previously, it has also been reported that the change in dipole moment obtained using Lippert-Mataga equation is larger than the values calculated by other methods [27, 30, 42].

The other important method to estimate the dipole moments depends on the empirical solvent polarity scale, $E_{T}^{N}[26,43]$. It correlates better with the solvatochromic data than the traditionally used bulk solvent polarity functions. In $E_{T}^{N}$, the error associated with the estimation of the Onsager cavity radius is reduced, and the empirical polarity scale also includes intermolecular interactions along with solvent polarity. Figures 6(a) and 6(b) are the graph of $\left(v_{a}-v_{f}\right)$ versus $E_{T}^{N}$ for LVF and NRF, respectively. The statistical results indicate that very high correlation coefficients $(R=0.91$ and 0.95$)$ were obtained for LVF and NRF, respectively. The good linearity of $E_{T}^{N}$ with the Stokes shift indicates the inclusion of both the solutesolvent interaction as well as H-bonding interaction in the empirical polarity scale. As it has been shown from the results of all models, the first excited state dipole moment is larger than the ground state dipole moment. The difference 
TABLE 3: Statistical analysis of the correlations of solvent spectral shifts of LVF and NRF.

\begin{tabular}{|c|c|c|c|}
\hline & Slope $\left(\mathrm{cm}^{-1}\right)$ & Intercept $\left(\mathrm{cm}^{-1}\right)$ & Correlation coefficient $\left(r^{2}\right)$ \\
\hline \multicolumn{4}{|c|}{ Bakhshiev's correlation } \\
\hline Levofloxacin & 5938 & 7707 & 0.93 \\
\hline Norfloxacin & 5899 & 7152 & 0.87 \\
\hline \multicolumn{4}{|c|}{ Kawski-Chamma-Viallet } \\
\hline Levofloxacin & 3463 & 58669 & 0.79 \\
\hline Norfloxacin & 4999 & 64848 & 0.86 \\
\hline \multicolumn{4}{|c|}{ Lippert-Mataga correlation } \\
\hline Levofloxacin & 19464 & 6858 & 0.87 \\
\hline Norfloxacin & 27749 & 4312 & 0.85 \\
\hline \multicolumn{4}{|c|}{ Reichardt correlation } \\
\hline Levofloxacin & 3140 & 10373 & 0.91 \\
\hline Norfloxacin & 4858 & 8738 & 0.95 \\
\hline
\end{tabular}

in dipole moment seen between two electronic states can be an indication of ICT [38].

In general, the dipole moments of the different states of a molecule are important parameters, which reveal information about the electronic and geometrical structures of the molecule. Investigating the ground and electronically excited state, dipole moments of a molecule provide elucidation of the excited state nature and reflect the charge distribution in the molecule. It is also used to predict the regions of electrophilic and nucleophilic reactivity in some photochemical reactions. Since both the pharmacological activities and the ground and excited state dipole moments of the molecule are sensitive to the molecular structure and geometry, small changes in dipole moments may be indicative of different pharmacological activities of the drugs. Therefore, investigation of spectral properties and dipole moment of LVF and NRF drug molecules in solvents is useful to understand features of the drugs systems.

4.3. Quantum Chemical Calculation. Figures 7(a)-7(c) show the HOMO-LUMO structures, the optimized structure with the dipole moment vector, and the total density matrix with the electrostatic potential map (TDM-ESP) for LFV and NRF using the semiempirical method MP6, DFT-B3LYP-321G, and DFT-B3LYP-6-31G, respectively. It shows the spatial distribution of the electron cloud in three dimensions. The three semiempirical methods provided similar results in which the electron clouds are mainly distributed on the molecular skeleton of the aromatic benzene ring of the drugs. Electron clouds are also present in the functional groups attached to the benzene ring.

The ground state dipole moments obtained from the semiempirical method PM6, DFT B3LYP-3-21G, and DFT B3LYP-6-31G are larger than the experimental results. For example, the ground state dipole moments obtained using DFT B3LYP-3-21G are $\mu_{g}=7.539 \mathrm{D}$ and $\mu_{g}=7.921 \mathrm{D}$ for LVF and NRF, respectively, and are larger than the experimental results as shown in Table 4 . The reason for such difference may be that the experimental method is affected by solvent and environmental effects (solute-solvent interaction), whereas the theoretical calculation is performed for a free molecule $[15,44]$. In addition, dipole moments obtained by the theoretical method are larger than experimental results due to theoretical dipole moments depending on charge densities obtained from eigenfunctions of the molecular orbital approximations. Also, the quantum chemical methods usually yield an exaggerated electrons distribution in molecules and make them more polar than in reality [30]. Recent work on 5-methyl-benzofuran-3-ylacetic acid hydrazide also indicated that the ground state dipole moment obtained by chemical calculation is larger than the excited state dipole moment [45]. On the other hand, the excited dipole moments obtained using the three methods are similar to the experimental value (5.970 and $7.160 \mathrm{D})$. The excited state dipole moment of LVF and NRF is $6.350,6.939$; 4.716, 6.20; and $5.277,6.813 \mathrm{D}$ using semiempirical method PM6, TD-SCF-DFT-B3LYP-3-21G, and TD-SCF-DFT-B3LYP-6-31G, respectively.

The HOMO-LUMO energy gap of LVF and NRF compounds, which indicates the chemical stability of the molecules in quantum chemistry, is calculated. A molecule with large HOMO-LUMO gaps is generally stable and unreactive, while ones with small gaps are generally reactive $[46,47]$. The HOMO-LUMO band gaps for LVF and NRF are $0.146,0.157$ and $0.151,0.162 \mathrm{eV}$ using DFT-B3LYP-6$31 \mathrm{G}$ and 3-21G, respectively. The HOMO-LUMO band gap energy of the two drugs is small compared to other aromatic compounds reported by [42], and this indicates that the drugs are highly reactive or that an electron of the HOMO orbital can easily be excited to the LUMO orbital $[33,48]$. A difference in the electronic distribution was also noticed on the HOMO-LUMO molecular orbital plots of LVF and NRF as shown in Figure 8. Unlike the experimental results, a higher electronic distribution was observed on the HOMO orbital level as compared to LUMO orbital level.

The electrostatic potential map plot of LVF and NRF shown in Figures 9(a), 9(b) makes it possible to estimate nucleophilic and electrophilic regions of the molecules. Identifying these nucleophilic and electrophilic regions is crucial to design nonlinear optical materials [49] and facilitates prediction of the site of attack in some photochemical reactions [30]. The electrostatic potential map of LVF and NRF is represented in red and blue colours. Blue colour represents a positive phase that corresponds to a nucleophilic region and red colour represents a negative 


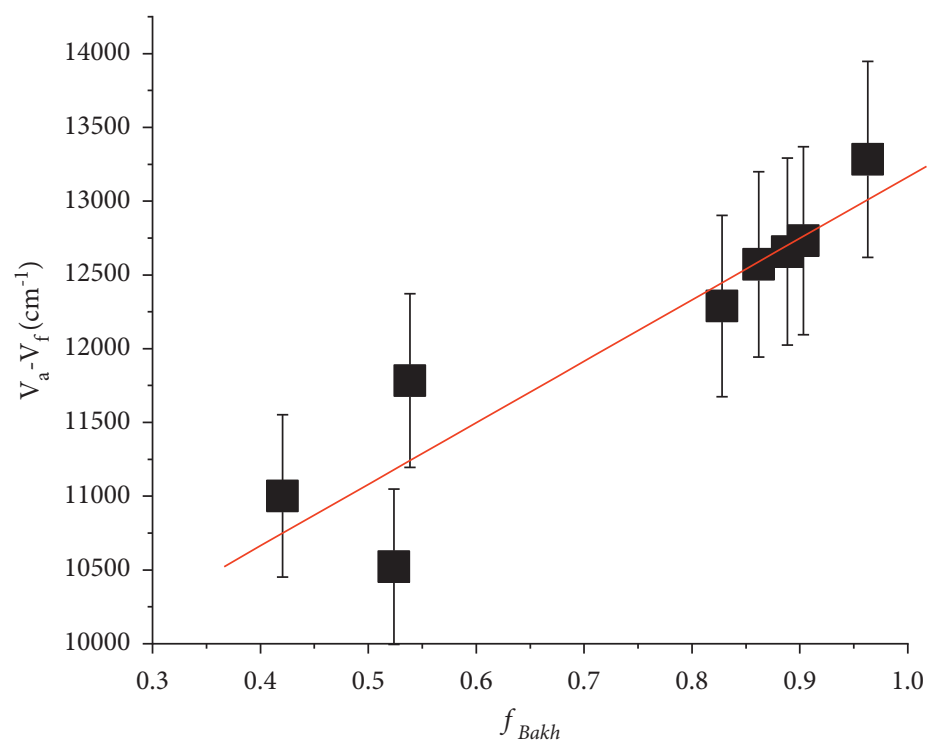

(a)

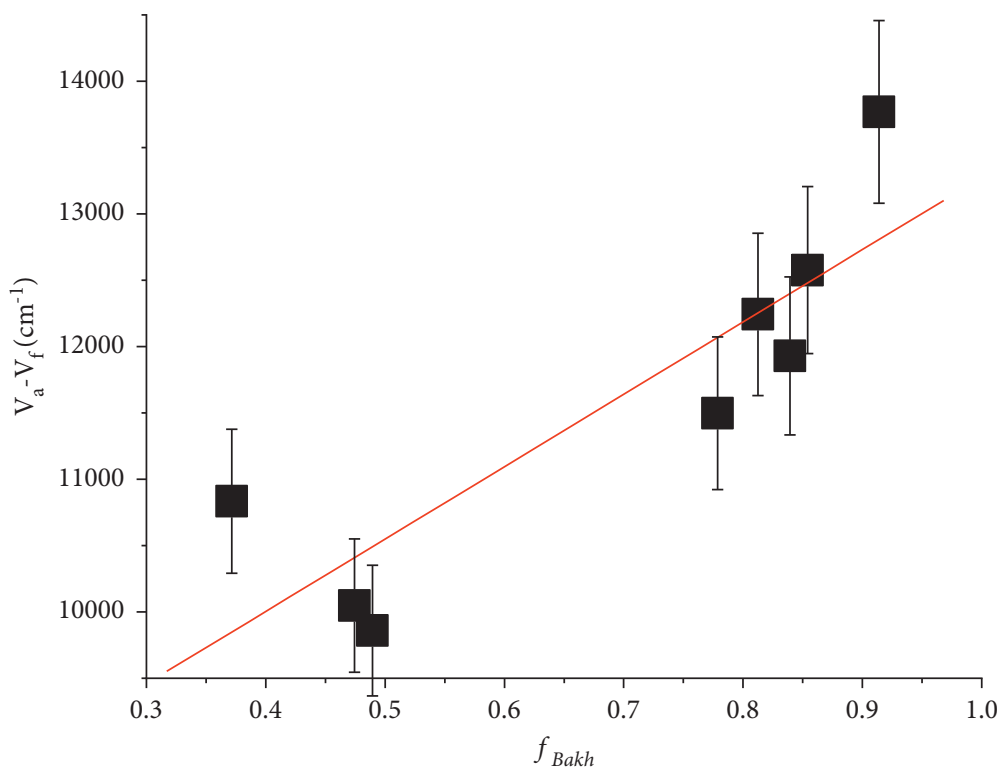

(b)

Figure 4: Plot of $v_{a}-v_{f}$ versus $f(\varepsilon, n)$ using Bakhshiev's equation for (a) LVF in different solvents (chloroform, dichloromethane, isopropanol, ethanol, methanol, distilled water, ethyl acetate, and ethyl glycol) and (b) NRF in different solvents (chloroform, dichloromethane, isopropanol, ethanol, methanol, distilled water, ethyl acetate, and ethyl glycol).

phase corresponds to an electrophilic region [35]. Oxygen creates an electron-rich region and the lowest electrostatic potential of the molecule, and nitrogen is relatively electron deficient for both LVF and NRF molecules. In addition, the $\mathrm{UV} / \mathrm{V}$ is absorption spectra, the excitation energies, and their corresponding oscillator strengths are determined using TD-SCF-DFT-B3LYP-6-31G since it determines these better than other methods $[50,51]$. The numerical values are shown in Table 5. The HOMO-LUMO structures and UV/Vis spectra are shown in Figure 10. 


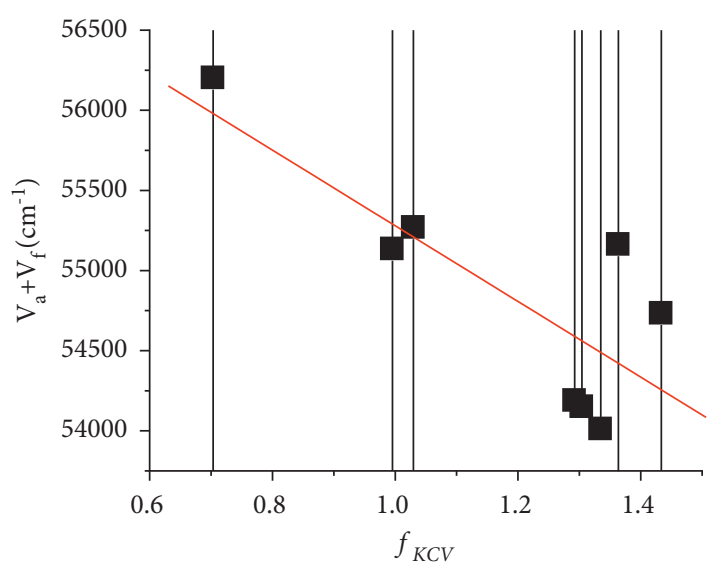

(a)

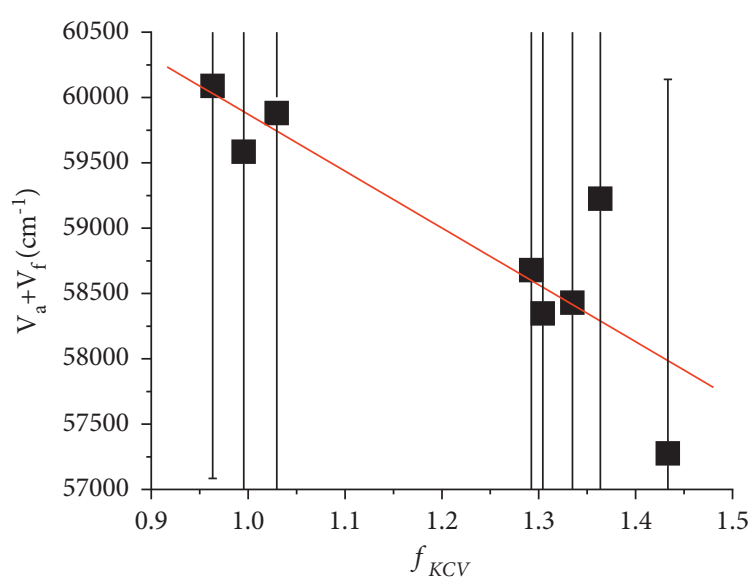

(b)

Figure 5: Plot of $v_{a}+v_{f}$ versus $f\left(\varepsilon_{r}, n\right)+2 g(n)$ using Kawski-Chamma-Viallet equation for (a) LVF in different solvents (chloroform, dichloromethane, isopropanol, ethanol, methanol, distilled water, ethyl acetate, and ethyl glycol) and (b) NRF in different solvents (chloroform, dichloromethane, isopropanol, ethanol, methanol, distilled water, ethyl acetate, and ethyl glycol).

TABLE 4: Calculated value of dipole moments in Debye (D) and Onsager cavity radius (a) obtained from experimental and theoretical work for LVF and NRF.

\begin{tabular}{lcccccccccc}
\hline Comp. & $a^{a}$ & $\mu_{g}^{b}$ & $\mu_{e}^{c}$ & $\mu_{e}^{d}$ & $\mu_{g}^{e}$ & $\mu_{e}^{f}$ & $\Delta \mu^{g}$ & $\Delta \mu^{h}$ & $\Delta \mu^{i}$ & $\left(\mu_{e} / \mu_{g}\right)^{j}$ \\
\hline LVF & 4.57 & 1.57 & 5.97 & 4.58 & 7.539 & 5.277 & 4.4 & 13.6 & 3.01 & 3.79 \\
NRF & 4.6 & 0.95 & 7.16 & 6.03 & 7.921 & 6.813 & 6.21 & 16.4 & 5.08 & 7.54 \\
\hline
\end{tabular}

a. Calculated value of Onsager cavity radius in angstrom. $b$. Experimental $\mu_{g}$ value calculated by (15). $c$. Experimental $\mu_{e}$ value calculated by (16). $d$. Experimental $\mu_{e}$ value calculated from empirical solvent polarity function. $e$. Theoretical $\mu_{g}$ values obtained by employing DFT B3LYP-6-21G. $f$. Theoretical $\mu_{e}$ values obtained by employing DFT B3LYP-6-31G. g. $\Delta \mu$ calculated from (15) and (16). $h$. $\Delta \mu$ calculated from (10). $i . \Delta \mu$ calculated from (20). j. Ratio of excited state and ground state dipole moment found by (17).

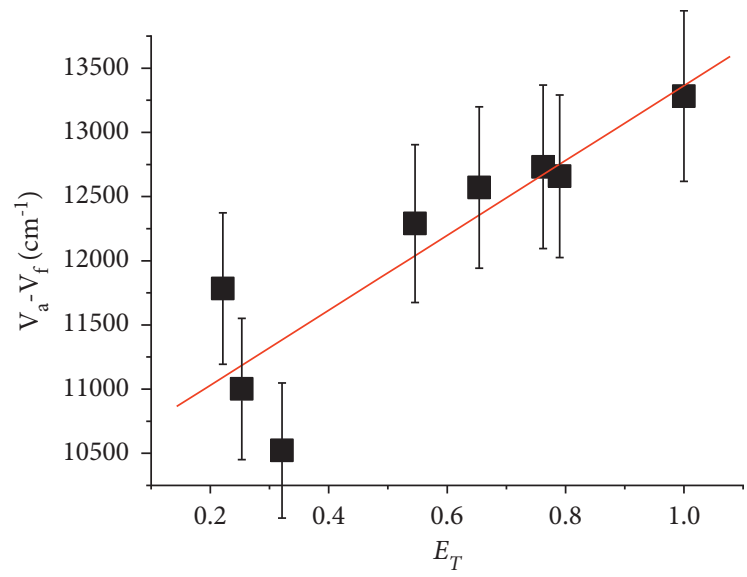

(a)

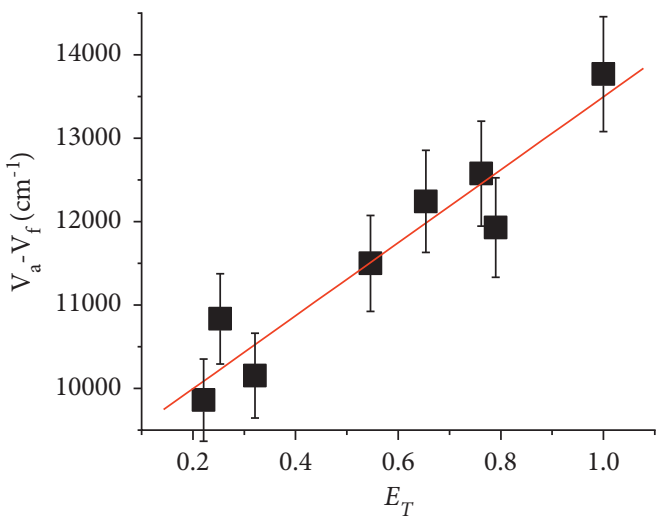

(b)

Figure 6: Plot of $v_{a}-v_{f}$ versus $E_{T}^{N}$ for Reichardt equation, (a) LVF in different solvents (chloroform, dichloromethane, isopropanol, ethanol, methanol, distilled water, ethyl acetate, and ethyl glycol) and (b) NRF in different solvents (chloroform, dichloromethane, isopropanol, ethanol, methanol, distilled water, ethyl acetate, and ethyl glycol). 


\begin{tabular}{|l|c|c|c|c|}
\hline Comp. & LUMO & Optimized & Structure & \\
\hline LVF & NRF & & &
\end{tabular}

(a)

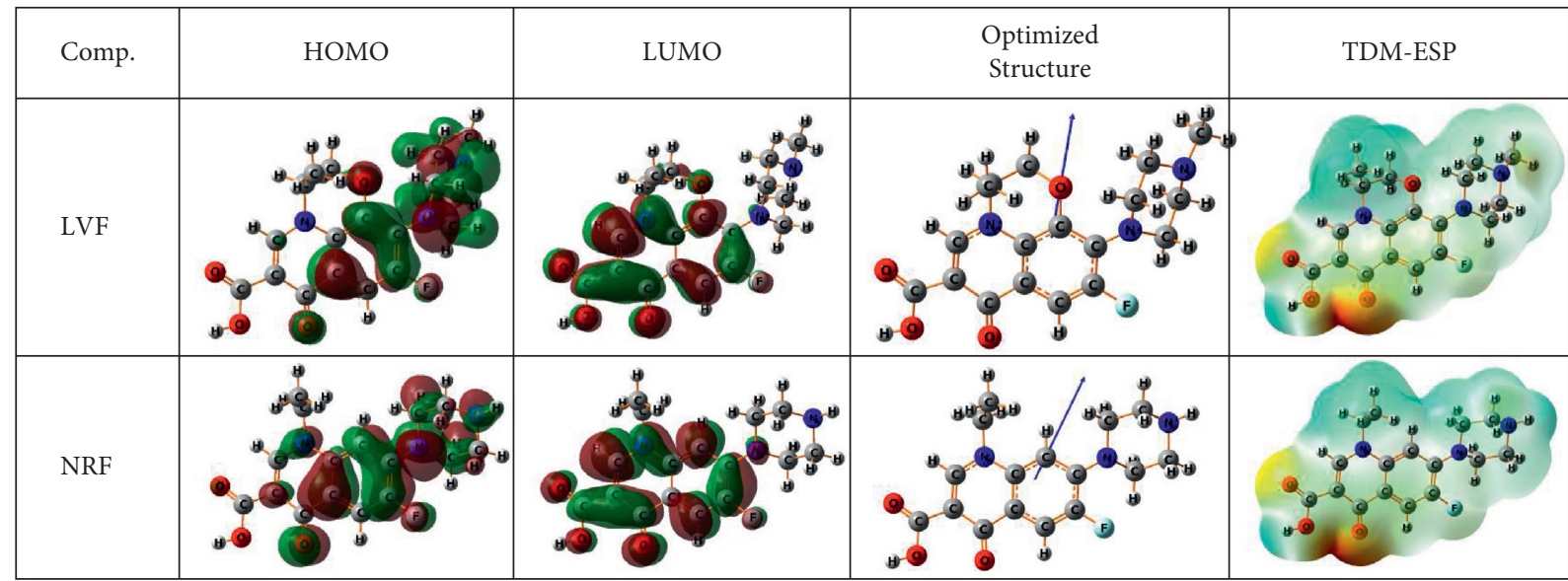

(b)

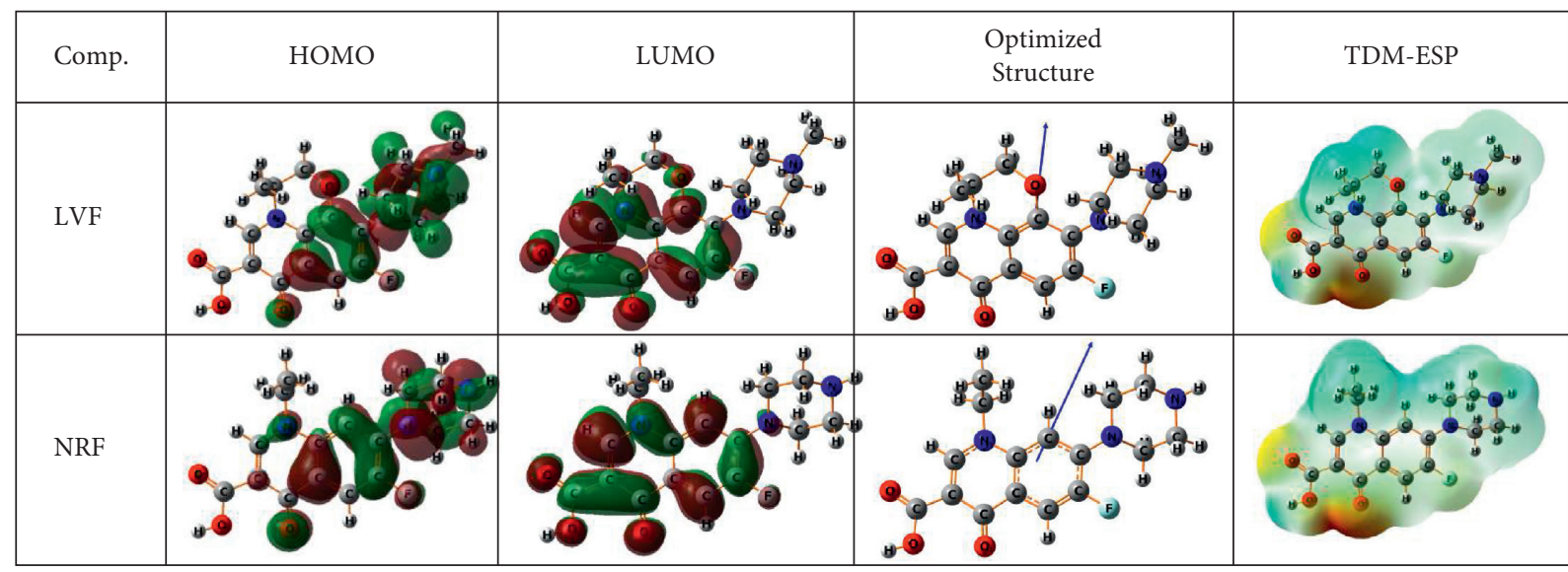

(c)

Figure 7: The HOMO-LUMO structures, optimized structure with dipole moment vector and total density matrix with electrostatic potential map (TDM-ESP) for LFV and NRF using (a) semiempirical method MP6, (b) DFT-B3LYP-3-21G, and (c). DFT-B3LYP-6-31G. 


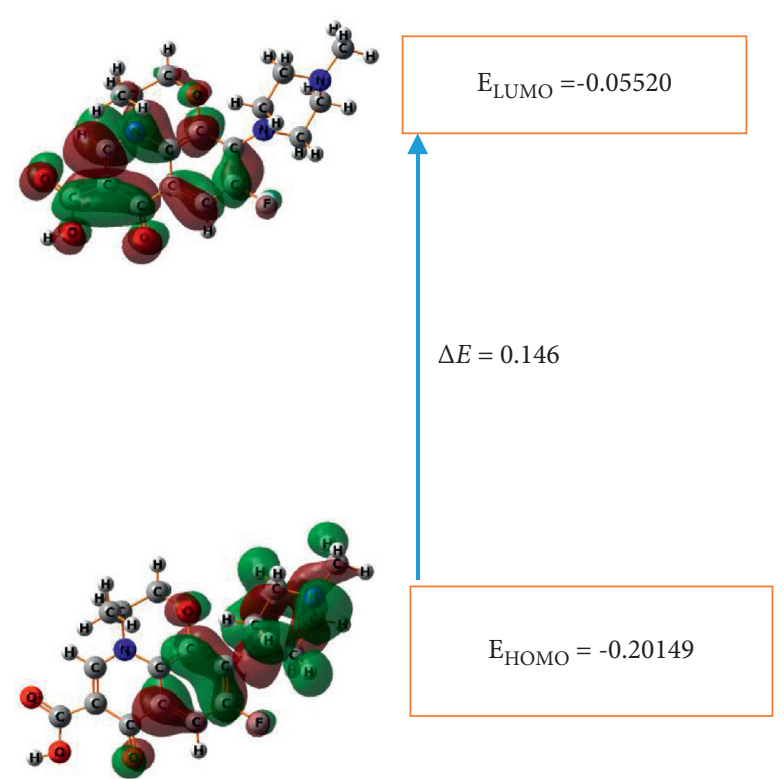

(a)
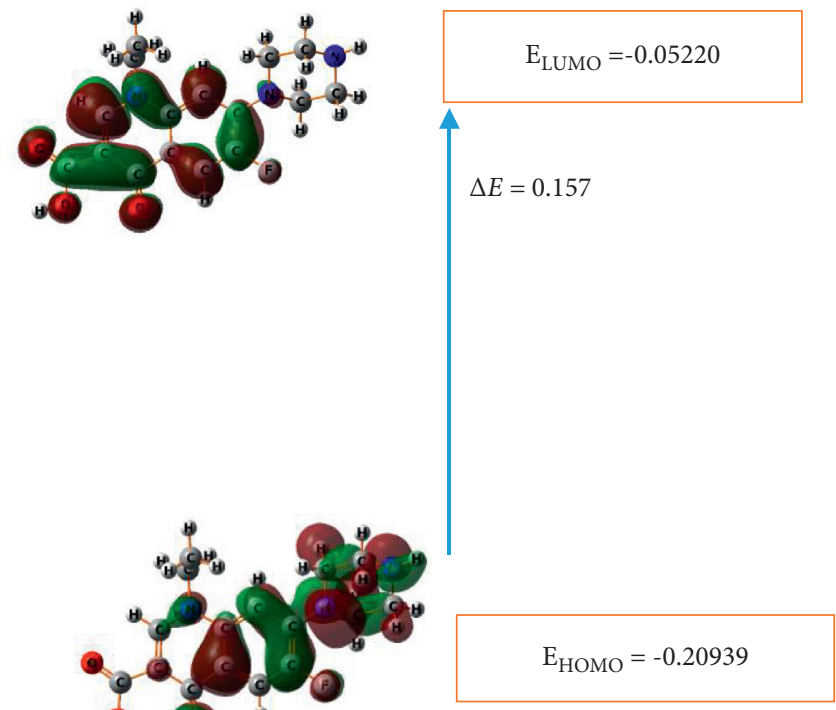

(b)

Figure 8: The HOMO-LUMO energy, band gap energy, and structure of (a) LVF and (b) NRF using DFT-B3LYP-6-31G.

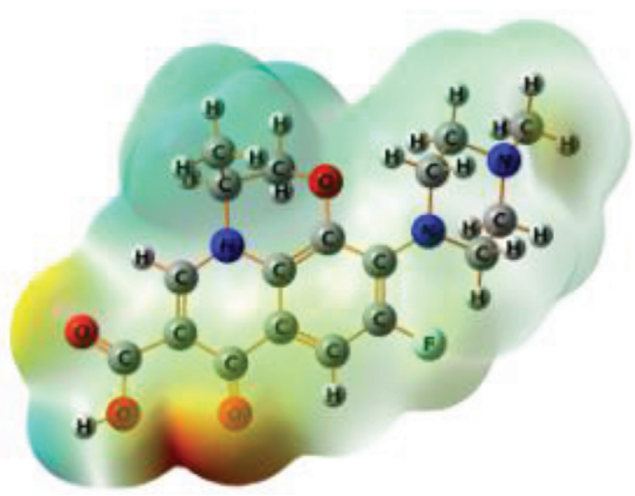

(a)

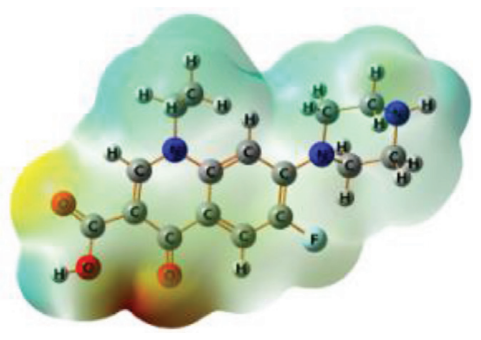

(b)

FIGURE 9: Total density matrix with electrostatic potential map (TDM-ESP) of (a) LVF and (b) NFX.

TABLE 5: HOMO-LUMO energy band gap, UV/Vis. absorption wavelengths and corresponding oscillator strengths determined using TDSCF-DFT-B3LYP-6-31G.

\begin{tabular}{lcccccccccc}
\hline Com. & Band gap $(\mathrm{eV})$ & $\lambda_{1}(\mathrm{~nm})$ & $\lambda_{2}(\mathrm{~nm})$ & $\lambda_{3}(\mathrm{~nm})$ & $E_{1}(\mathrm{eV})$ & $E_{2}(\mathrm{eV})$ & $E_{3}(\mathrm{eV})$ & $\mathrm{S}_{1}$ & $\mathrm{~S}_{2}$ & $\mathrm{~S}_{3}$ \\
\hline LVF & 0.123 & 416.4 & 394.0 & 386.3 & 2.9773 & 3.1467 & 3.2098 & 0 & 0.0262 & 0.0003 \\
NRF & 0.141 & 390.2 & 360.2 & 359.1 & 3.1773 & 3.4424 & 3.4529 & 0.0003 & 0.0118 & 0.0609 \\
\hline
\end{tabular}

$E_{1}$ : excited state 1 energy and corresponding wavelength, $\lambda_{1}$; oscillator strength, $\mathrm{S}_{1}$. $E_{2}$ : excited state 2 energy and corresponding wavelength, $\lambda_{2}$; oscillator strength, $S_{2} . E_{3}$ : excited state 3 energy and corresponding wavelength, $\lambda_{3}$ and oscillator strength, $S_{3}$. 


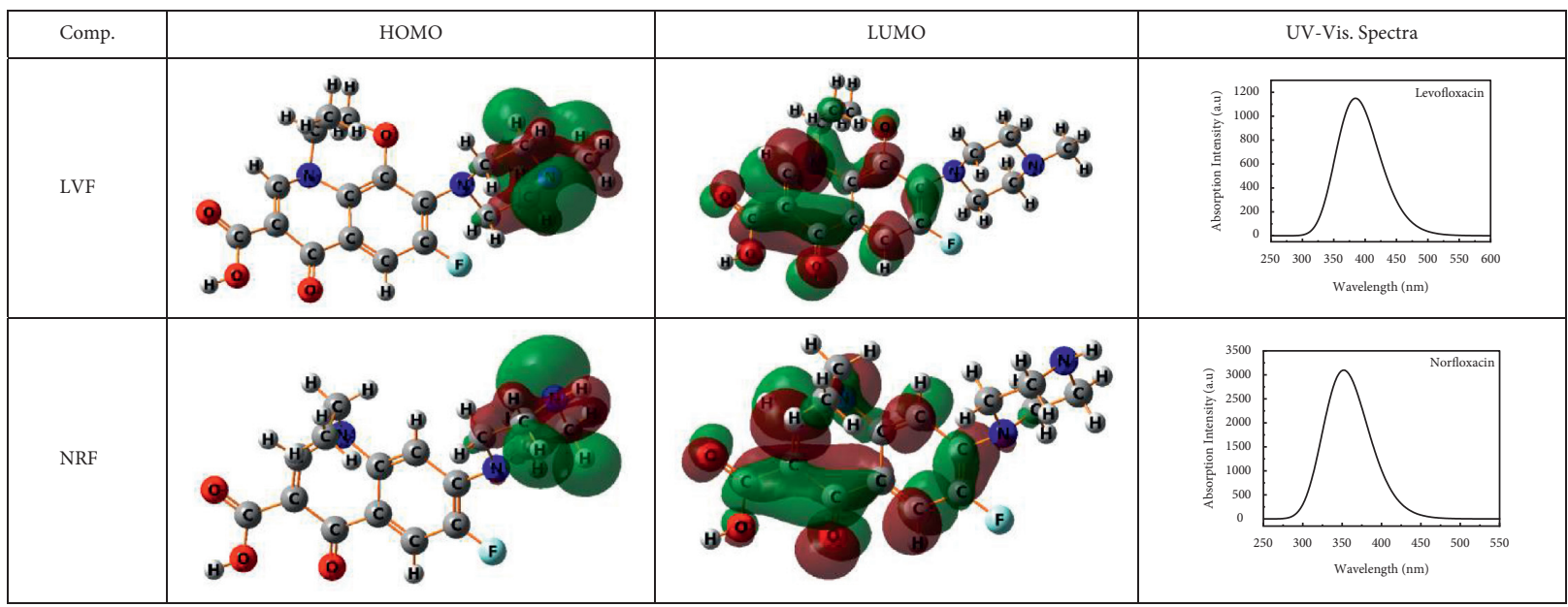

FIGURE 10: HOMO-LUMO structures and UV/Vis spectra of LVF and NRF determined using TD-SCF-DFT-B3LYP-6-31G.

\section{Conclusion}

The effect of solvent polarity on the absorption and emission spectra of LVF and NRF were investigated to estimate dipole moments. The results indicate that the emission spectra of both compounds are more strongly affected than the electronic absorption spectra. The dipole moments of LVF and NRF were estimated by Lippert-Mataga, Bakhshiev's, Kawski-Chamma-Viallet, and Reichardt methods. The excited state dipole moments calculated from experimental results are larger than the ground state dipole moments indicating that the probe compounds are significantly more polar in the excited state than the ground state, and it can also be an indication of ICT. The discrepancy in the ground and excited state dipole moment obtained from different equation are due to different assumption and simplifications that are used in each method. The ground and excited state dipole moments of NRF are larger than that of LVF, and this result may be due to the structural differences and Onsager cavity radius of the two drugs. Computational analysis was performed by Gaussian 09 using DFT methods at B3LYP-3-21G and B3LYP-6-31G level of theory and the semiempirical MP6 method. The calculated HOMO-LUMO energy band gap obtained by all methods is small, and this indicates that both compounds are highly reactive. Larger dipole moments were obtained from computational work than from the experimental results, due to the absence of solvent effects. In general, the spectral properties observed, the values of dipole moments, and electronic structures of LVF and NRF of antibiotic drugs in polar and nonpolar solvents provide important information about charge distribution and solute-solvent interactions, which may be useful in the studying of these molecules in biological systems.

\section{Data Availability}

The data used to support the findings of this study are included within the article. The data are generated during the study in the laboratory, and they are described in each figure and table in this article.

\section{Conflicts of Interest}

The authors declare that they have no conflicts of interest.

\section{Acknowledgments}

The authors wish to acknowledge Adama Science and Technology University and Ministry of Innovation and Technology for financial support to carry out this research. The authors also acknowledge and thank Dr. S. M. Hanagodimath for his contribution doing computational work.

\section{References}

[1] P. C. Sharma, A. Jain, and S. Jain, "Fluoroquinolone antibacterials: a review on chemistry, microbiology and therapeutic prospects," Acta poloniae pharmaceutica, vol. 66, pp. 587-604, 2009.

[2] R. Kian, M. S. Zakerhamidi, A. N. Shamkhali, and E. Kashani, "Study of the variation of intra/intermolecular interactions and configuration of a group of enone anticancer drugs as a result of solvation," Journal of Molecular Liquids, vol. 274, pp. 1-14, 2019.

[3] G. Viola, A. Salvador, L. Cecconet et al., "Photophysical properties and photobiological behavior of amodiaquine, primaquine and chloroquine," Photochemistry and Photobiology, vol. 83, no. 6, pp. 1415-1427, 2007.

[4] G. Sárközy, "Quinolones: a class of antimicrobial agents," Veterinarni Medicina (Praha), vol. 46, pp. 257-274, 2001.

[5] R. Kian, M. S. Zakerhamidi, A. N. Shamkhali, R. TeimuriMofrad, and K. Rahimpour, "Media and solute-solvent interaction effects on the photo-physical behavior of some drugs of $4 \mathrm{H}$-pyran derivatives," Journal of Molecular Liquids, vol. 238, pp. 508-517, 2017.

[6] R. Kian, M. S. Zakerhamidi, A. Ranjkesh et al., "Investigation of the spectroscopic features along with the media polarity effect in some symmetrical disc-shaped liquid crystals," Journal of Molecular Liquids, vol. 309, Article ID 113226, 2020.

[7] G. Viola, L. Facciolo, M. Canton et al., "Photophysical and phototoxic properties of the antibacterial fluoroquinolones levofloxacin and moxifloxacin," Chemistry \& Biodiversity, vol. 1, no. 5, pp. 782-801, 2004. 
[8] B. Siddlingeshwar, S. M. Hanagodimath, E. M. Kirilova, and G. K. Kirilov, "Photophysical characteristics of three novel benzanthrone derivatives: experimental and theoretical estimation of dipole moments," Journal of Quantitative Spectroscopy and Radiative Transfer, vol. 112, no. 3, pp. 448-456, 2011.

[9] R. Kian, S. M. Seyed Ahmadian, and M. S. Zakerhamidi, "Photo-physical properties of different types of vitamin A in solvent media," Journal of Molecular Structure, vol. 1080, pp. 8-13, 2015.

[10] M. S. Zakerhamidi, L. Zare Haghighi, and S. M. Seyed Ahmadian, "Photo-physical and interactional behavior of two members of group B vitamins in different solvent media," Journal of Molecular Structure, vol. 1144, pp. 265-272, 2017.

[11] R. Kian, M. S. Zakerhamidi, A. N. Shamkhali, and P. Nesari, "The interactional behaviors and photo-physical properties of two triarylmethane drugs in solvent media," Journal of Molecular Liquids, vol. 225, pp. 653-661, 2017.

[12] M. Khadem Sadigh, M. S. Zakerhamidi, S. M. Seyed Ahmadian, M. Johari-Ahar, and L. Zare Haghighi, "Environment and solute-solvent interaction effects on photophysical behaviors of folic acid and folinic acid drugs," Journal of Molecular Structure, vol. 1125, pp. 177-185, 2016.

[13] M. Khadem Sadigh, M. S. Zakerhamidi, S. M. Seyed Ahmadian, M. Johari-Ahar, and L. Zare Haghighi, "Environment effect on spectral and charge distribution characteristics of some drugs of folate derivatives," Spectrochimica Acta Part A: Molecular and Biomolecular Spectroscopy, vol. 171, pp. 10-17, 2017.

[14] D. D. Thiaré, A. Khonté, A. Diop et al., "Determination of ground and excited state dipole moments of amino-benzimidazole by solvatochromic shift methods and theoretical calculations," Journal of Molecular Liquids, vol. 211, pp. 640-646, 2015.

[15] I. Sidir and Y. Gülseven Sidir, "Estimation of ground and excited state dipole moments of oil red o by solvatochromic shift methods," Spectrochimica Acta - Part A Molecular and Biomolecular Spectroscopy, vol. 135, pp. 560-567, 2015.

[16] V. R. Desai, A. H. Sidarai, S. M. Hunagund et al., "Steady state absorption and fluorescence study: estimation of ground and excited state dipole moments of newly synthesized pyridazin$3(2 \mathrm{H})$-one derivatives," Journal of Molecular Liquids, vol. 223, pp. 141-149, 2016.

[17] U. S. Raikar, C. G. Renuka, Y. F. Nadaf, B. G. Mulimani, A. M. Karguppikar, and M. K. Soudagar, "Solvent effects on the absorption and fluorescence spectra of coumarins 6 and 7 molecules: determination of ground and excited state dipole moment," Spectrochimica Acta Part A: Molecular and Biomolecular Spectroscopy, vol. 65, no. 3-4, pp. 673-677, 2006.

[18] İ. Sidır and Y. G. Sidır, "Ground state and excited state dipole moments of 6,8-diphenylimidazo[1,2- $\alpha]$ pyrazine determined from solvatochromic shifts of absorption and fluorescence spectra," Spectrochimica Acta Part A: Molecular and Biomolecular Spectroscopy, vol. 79, pp. 1220-1225, 2011.

[19] A. Kawski, P. Bojarski, and B. Kukliński, "Excitation wavelength dependence of ACRYLODAN fluorescence spectra in some polar solvents," Zeitschrift Für Naturforschung A, vol. 57, no. 9-10, pp. 94-97, 2002.

[20] L. Bilot and A. Kawski, "Zur theorie des einflusses von lösungsmitteln auf die elektronenspektren der moleküle," Zeitschrift Für Naturforschung A, vol. 17, no. 7, pp. 621-627, 1962.

[21] E. Lippert, "Dipolmoment und Elektronenstruktur von angeregten molekülen," Zeitschrift Für Naturforschung A, vol. 10, no. 7, pp. 541-545, 1955.
[22] N. Mataga, Y. Kaifu, and M. Koizumi, "Solvent effects upon fluorescence spectra and the dipolemoments of excited molecules," Bulletin of the Chemical Society of Japan, vol. 29, no. 4, pp. 465-470, 1956.

[23] N. G. Bakhshiev, M. I. Knyazhanskii, V. I. Minkin, O. A. Osipov, and G. V. Saidov, "Experimental determination of the dipole moments of organic molecules in excited electronic states," Russian Chemical Reviews, vol. 38, no. 9, pp. 740-754, 1969.

[24] P. Suppan, "Excited-state dipole moments from absorption/ fluorescence solvatochromic ratios," Chemical Physics Letters, vol. 94, no. 3, pp. 272-275, 1983.

[25] J. T. Edward, "Molecular volumes and the Stokes-Einstein equation," Journal of Chemical Education, vol. 47, no. 4, pp. 261-270, 1970.

[26] C. Reichardt, "Solvatochromic dyes as solvent polarity indicators," Chemical Reviews, vol. 94, no. 8, pp. 2319-2358, 1994.

[27] M. Ravi, T. Soujanya, A. Samanta, and T. P. Radhakrishnan, "Excited-state dipole moments of some Coumarin dyes from a solvatochromic method using the solvent polarity parameter," Journal of the Chemical Society, Faraday Transactions, vol. 91, no. 17, pp. 2739-2742, 1995.

[28] M. J. Frisch, G. W. Trucks, H. B. Schlegel et al., Gaussian 09, Revision B.01, Gaussian 09, Revis. B.01, Gaussian, Inc., London, UK, 2009.

[29] E. Bozkurt, H. İ. Gül, and M. Tuğrak, "Investigation of solvent effect on photophysical properties of some sulfonamides derivatives," Turkish Journal of Chemistry, vol. 41, pp. 282-293, 2017.

[30] S. R. Manohara, V. U. Kumar, L. Shivakumaraiah, and L. Gerward, "Estimation of ground and excited-state dipole moments of 1,2 -diazines by solvatochromic method and quantum-chemical calculation," Journal of Molecular Liquids, vol. 181, pp. 97-104, 2013.

[31] J. Basavaraja, H. M. Suresh Kumar, S. R. Inamdar, and M. N. Wari, "Estimation of ground and excited state dipole moment of laser dyes C504T and C521T using solvatochromic shifts of absorption and fluorescence spectra," Spectrochimica Acta Part A: Molecular and Biomolecular Spectroscopy, vol. 154, pp. 177-184, 2016.

[32] A. Al Sabahi, S. N. Al, F. O. Suliman, and S. M. Al Kindy, "Photophysical and theoretical studies on the solvatochromic effects and dipole moments evaluation of substituted 1phenyl-3-naphthyl-5- (4-ethyl benzoate) -2-pyrazoline," p. $307,2020$.

[33] M. Sharma, U. Pal, M. Kumari et al., "Effect of solvent on the photophysical properties of isoxazole derivative of curcumin: a combined spectroscopic and theoretical study," Journal of Photochemistry and Photobiology A: Chemistry, vol. 410, Article ID 113164, 2021.

[34] M. C. Cuquerella, M. A. Miranda, and F. Bosca, "Role of excited state intramolecular charge transfer in the photophysical properties of norfloxacin and its derivatives," The Journal of Physical Chemistry A, vol. 110, no. 8, pp. 2607-2612, 2006.

[35] M. K. Patil, M. G. Kotresh, and S. R. Inamdar, "A combined solvatochromic shift and TDDFT study probing solute-solvent interactions of blue fluorescent alexa fluor 350 dye: evaluation of ground and excited state dipole moments," Spectrochimica Acta Part A: Molecular and Biomolecular Spectroscopy, vol. 215, pp. 142-152, 2019.

[36] S. A. Patil, P. S. Kadolkar, M. N. Wari, and S. R. Inamdar, "Photo-physical study of coumarins in aqueous organic solvents: an experimental and theoretical approach," Materials Today Communications, vol. 29, Article ID 102733, 2021. 
[37] S. Walki, G. H. Malimath, K. M. Mahadevan et al., "Synthesis, spectroscopic properties, and DFT correlative studies of 3,3'carbonyl biscoumarin derivatives," Journal of Molecular Structure, vol. 1243, Article ID 130781, 2021.

[38] N. Fatma, M. S. Mehata, N. Pandey, and S. Pant, "Experimental and theoretical interpretations of spectral behavior of 6-methoxyflavone," Journal of Photochemistry and Photobiology A: Chemistry, vol. 404, Article ID 112945, 2021.

[39] B. Siddlingeshwar and S. M. Hanagodimath, "Estimation of the ground and the first excited singlet-state dipole moments of 1,4-disubstituted anthraquinone dyes by the solvatochromic method," Spectrochimica Acta Part A: Molecular and Biomolecular Spectroscopy, vol. 75, no. 4, pp. 1203-1210, 2010.

[40] A. Benazzouz, M. Makhloufi-Chebli, S. M. Hamdi, B. Boutemeur-Kheddis, A. M. S. Silva, and M. Hamdi, "Study of novel fluorescent coumarin-3,4-dihydropyrimidin-2 $(1 \mathrm{H})$ ones dyads. estimation of ground- and excited-state dipole moments from a solvatochromic shift," Journal of Molecular Liquids, vol. 219, pp. 173-179, 2016.

[41] M. Bratkowska, T. Wybranowski, and I. Ho, Experimental and Theoretical Insight into Spectroscopic Properties and for Future Applications in Biochemistry, p. 314, 2020.

[42] A. Belay, E. Libnedengel, H. K. Kim, and Y.-H. Hwang, "Effects of solvent polarity on the absorption and fluorescence spectra of chlorogenic acid and caffeic acid compounds: determination of the dipole moments," Luminescence, vol. 31, no. 1, pp. 118-126, 2016.

[43] G. V. Muddapur, N. R. Patil, S. S. Patil, R. M. Melavanki, and R. A. Kusanur, "Estimation of ground and excited state dipole moments of aryl boronic acid derivative by solvatochromic shift method," Journal of Fluorescence, vol. 24, no. 6, pp. 1651-1659, 2014.

[44] I. Sidir, Y. G. Sidir, H. Berber, and G. Türkołlu, "Specific and non-specific interaction effect on the solvatochromism of some symmetric (2-hydroxybenzilydeamino)phenoxy Schiff base derivatives," Journal of Molecular Liquids, vol. 215, pp. 691-703, 2016.

[45] C. V. Maridevarmath, L. Naik, V. S. Negalurmath, M. Basanagouda, and G. H. Malimath, "Synthesis, photophysical, DFT and solvent effect studies on biologically active benzofuran derivative: (5-methyl-benzofuran-3-yl)-acetic acid hydrazide," Chemical Data Collections, vol. 21, 2019.

[46] A. C. Morosanu, D. G. Dimitriu, and D. O. Dorohoi, "Excited state dipole moment of the fluorescein molecule estimated from electronic absorption spectra," Journal of Molecular Structure, vol. 1180, pp. 723-732, 2019.

[47] L. M. Ivan, D. G. Dimitriu, A. Gritco-Todirascu, A. C. Morosanu, D. O. Dorohoi, and C. Cheptea, "Excited state dipole moment of two pyridazinium-p-nitro-phenacylids estimated from solvatochromic study," Spectroscopy Letters, vol. 53, no. 1, pp. 1-11, 2020.

[48] T. Uv, E. B. V All, and I. Salicylates, "Experimental and theoretical investigation of the molecular, electronic structure and solvatochromism of phenyl salicylate," External Electric Field Effect on the Electronic Structure, vol. 1147, pp. 206-216, 2017.

[49] K. B. Akshaya, A. Varghese, P. L. Lobo, R. Kumari, and L. George, "Synthesis and photophysical properties of a novel phthalimide derivative using solvatochromic shift method for the estimation of ground and singlet excited state dipole moments," Journal of Molecular Liquids, vol. 224, pp. 247-254, 2016.

[50] P. Krawczyk, "Time-dependent density functional theory calculations of the solvatochromism of some azo sulfonamide fluorochromes," Journal of Molecular Modeling, vol. 21, no. 5, p. 118, 2015.

[51] A. R. Allouche, "Gabedit--a graphical user interface for computational chemistry softwares," Journal of Computational Chemistry, vol. 32, no. 1, pp. 174-182, 2011. 